\title{
BIOLOGIE ET ÉCOLOGIE DE BARBUS HOLOTAENIA, BOULENGER, 1904, DU BASSIN DE LA RIVIĖRE LUKI (ZAÏRE).
}

\section{S. MUTAMBUE}

\author{
Département de Biologie Chimie, Institut Supérieur Pédagogique Mbanza-Ngungu, \\ B.P. 127, Mbanza-Ngungu, République du Zaïre.
}

\section{RÉSUMÉ}

Barbus holotaenia est un petit Cyprinidae (142,5 mm. LS) largement répandu dans la région guinéenne. Son régime alimentaire est composé principalement d'invertébrés aquatiqués et d'insectes terrestres, en saison des pluies; de crevettes et d'écailles, en saison sèche. La taille à la première maturation est de $42,5 \mathrm{~mm}$ LS chez les mâles et de $37,5 \mathrm{~mm}$ LS chez les femelles. La vitellogenèse et la ponte se déroulent en saison des pluies. La fécondité individuelle partielle varie entre 5 et 18 oeufs par grammé de la masse du corps de la femelle. Le coefficient de condition est indépendant de la taille; les valeurs les plus élevées s'observent en saison des pluies. La croissance de l'espèce est analysée par un modèle d'estimation rapide de la croissance des poissons d'eau douce africains.

Mots-clés : Zaïre, Barbus holotaenia, biologie, écologie, croissance, régime alimentaire, fécondité, coefficient de condition.

\section{BIOLOGY AND ECOLOGY OF BARBUS HOLOTAENIA, BOULENGER, 1904, FROM THE LUKI RIVER BASIN (ZAIIRE).}

\begin{abstract}
Barbus holotaenia is a small Cyprinidae (142,5 SL mm) widely distributed in the Guinean region. Its diet is mainly composed by aquatic benthic and terrestrial insects in the rainy season and by shrimps and fish scale in the dry season. The length at first maturity is $42,5 \mathrm{~mm}$ LS for males and $37,5 \mathrm{~mm}$ SL for females. Vitellogenesis and breeding take place in the rainy season. The partial individual fecundity varies between 5 and 18 eggs per gram of female weight. The condition coefficient is independent of the length. The highest values are observed in the rainy season. The species growth is analysed by a short cut model for estimation of freshwater fish growth of Africa.
\end{abstract}

Key-words : Zaïre, Barbus holotaenia, biology, ecology, growth, diet, fecundity, condition coefficient.

\section{INTRODUCTION}

Barbus holotaenia est un petit Cyprinidae rhéophile qui vit régulièrement et souvent en grand nombre dans de nombreux fleuves, rivières, ruisseaux et marigots de la région guinéenne (BOULENGER, 1909-1916 ; POLL, 1941, 1960, 1967 ; POLL et GOSSE, 1963 ; GOSSE, 1963 ; MATTHES, 1964 ; BLACHE, 1964 ; BANISTER, 1973 ; GERY, 1965 ; MAHNERT et GERY, 1982 ; MUTAMBUE, 1984, 1985, 1992).

Dans le bassin de la rivière Luki, il est absent des ruisseaux et des zones de sources, rare dans les zones inférieures et très abondant dans les cours moyens à fond garni de sables et/ou de sables associés aux cailloux et/ou aux galets. Des études de répartition spatiale ont montré que les adultes vivent en pleine eau, se tiennent dans le courant et se déplacent par bancs alors que les jeunes se cantonnent le long des rives où leur présence est corrélée à l'existence des caches (MUTAMBUE, 1992 ; CUGNY et MUTAMBUE, 1991). 
Pour MAHNERT et GERY (1982), les caractères méristiques, les proportions et la forme du maxillaire-prémaxillaire des poissons rencontrés dans les différents milieux aquatiques africains ne présentent pas de différence avec les exemplaires qui ont servi à la description originale. Cependant, des différences de coloration suivant les localités et même suivant l'âge ont été évoquées à maintes reprises par de nombreux auteurs (BOULENGER, 1911 ; FOWLER, 1930 ; DAGET, 1961 ; ROMAN, 1971 ; MAHNERT et GERY, 1982).

Dans ces milieux aquatiques, $B$. holotaenia constitue une part importante des proies consommées par les ichtyophages tels Opsaridium chrystyi (BOULENGER, 1920), Hydrocynus forskalii (CUVIER, 1819), Schilbe (Eutropius) niloticus (RÜPPEL, 1829), Schilbe (Eutropius) grenfelli (BOULENGER, 1900), Schilbe (Schilbe) mystus (LINNAEUS, 1758), Schilbe (Schilbe) uranoscopus (RÜPPEL, 1832) ou Bagrus bajad (FORSSKAL, 1775).

Sa systématique ayant fait l'objet de plusieurs travaux (voir C.L.O.F.F.A., 1984), nous avons axé notre attention sur sa biologie et son écologie.

En dehors de son intérêt théorique, cette étude ainsi que celles d'autres espèces pourraient contribuer largement à leur conservation lorsqu'on sait qu'on ne peut à l'heure actuelle gérer cette faune, qui fait partie intégrante de la Réserve de la Biosphère de la Luki, sans appel à la connaissance qui, pour reprendre l'expression de LEFEUVRE et al. (1979), constitue la première étape d'une politique de conservation à court et/ou à long terme.

\section{MILIEUX D'ÉTUDE}

Cette étude porte sur les poissons récoltés dans le bassin de la rivière Luki couvrant une superficie de $756 \mathrm{~km}^{2}$ environ et situé entre $12^{\circ} 53^{\prime}$ et $13^{\circ} 17^{\prime} \mathrm{E}$ et $5^{\circ} 30^{\prime}$ et $5^{\circ} 47^{\prime} \mathrm{S}$ (Fig. 1 ).

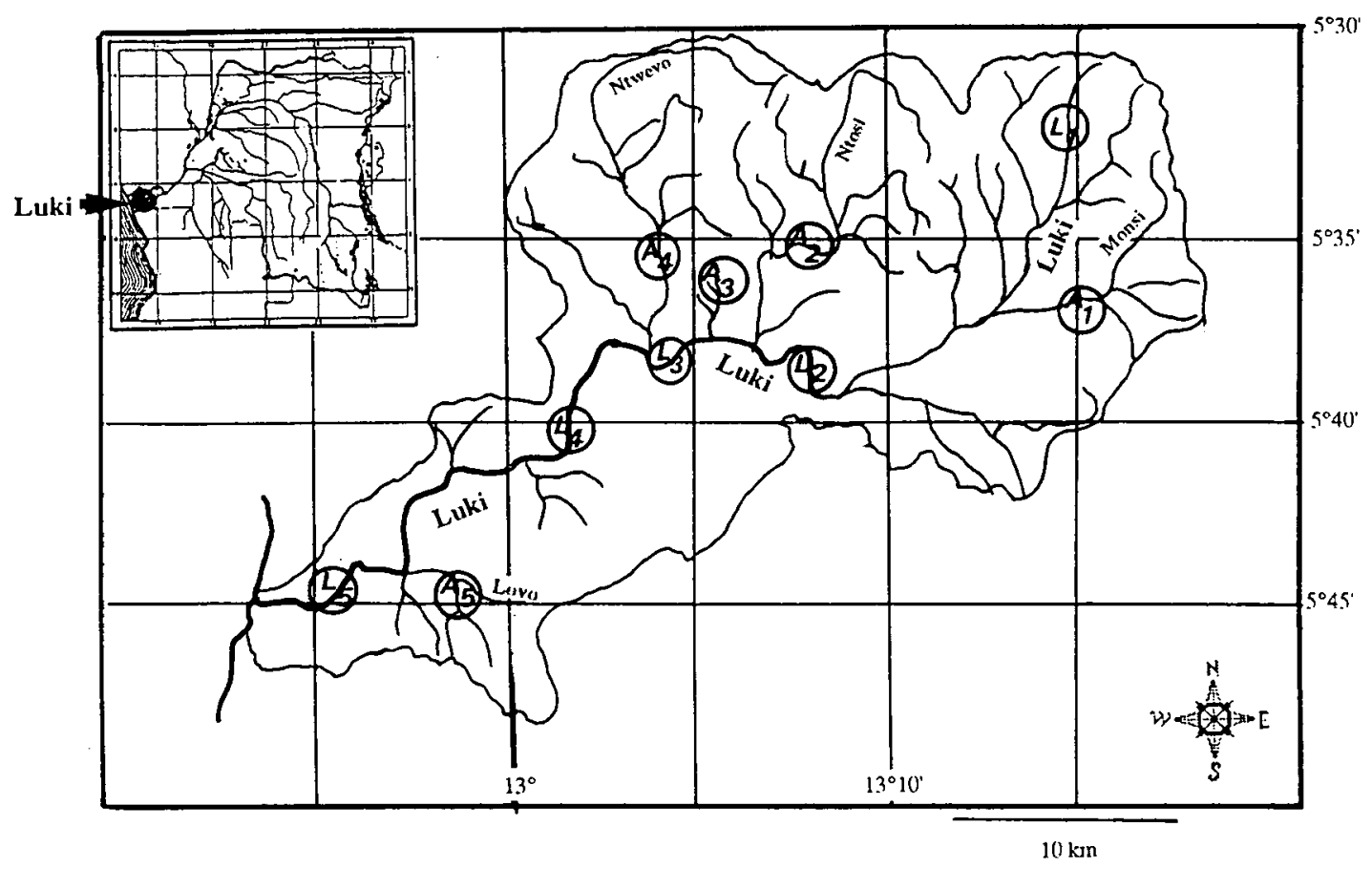

Figure 1 : Situation géographique du bassin de la Luki.

Figure 1 : Geographical situation of the Luki river basin. 
La rivière principale (la Luki) est alimentée par un important réseau secondaire. Le réseau tertiaire constitué par de nombreux ruisselets et rigoles, des ruisseaux et des torrents, situés presque tous en contrebas des plateaux, est également considérable. Ces différents cours d'eau sont presque tous parallèles et suivent plus ou moins la direction tectonique de la région.

De par sa situation géographique, ce bassin bénéficie d'un climat à caractère tropical marqué par l'alternance de la saison des pluies et de la saison sèche qui, selon AUBREVILLE (1949), coïncide avec l'extension septentrionale de l'alizé austral et du courant côtier, dit de Bengwela (Fig. 2). Les précipitations y sont irrégulières et les observations effectuées ces trente dernières années fixent les caractéristiques suivantes :

- précipitations annuelles moyennes : $1123,62 \mathrm{~mm}$

— températures annuelles moyennes : $23,8^{\circ} \mathrm{C}$.

Les caractéristiques générales du régime hydrologique de différents cours d'eau rencontrés dans ce bassin dépendent des facteurs climatiques et plus particulièrement du régime pluviométrique ainsi que du régime bimodal du fleuve Zaïre qui, lors des crues, empêche l'écoulement de ses affluents et sous-affluents et crée un courant à contresens. L'action de ces différents paramètres se traduit par deux crues et deux décrues (Fig. 2).

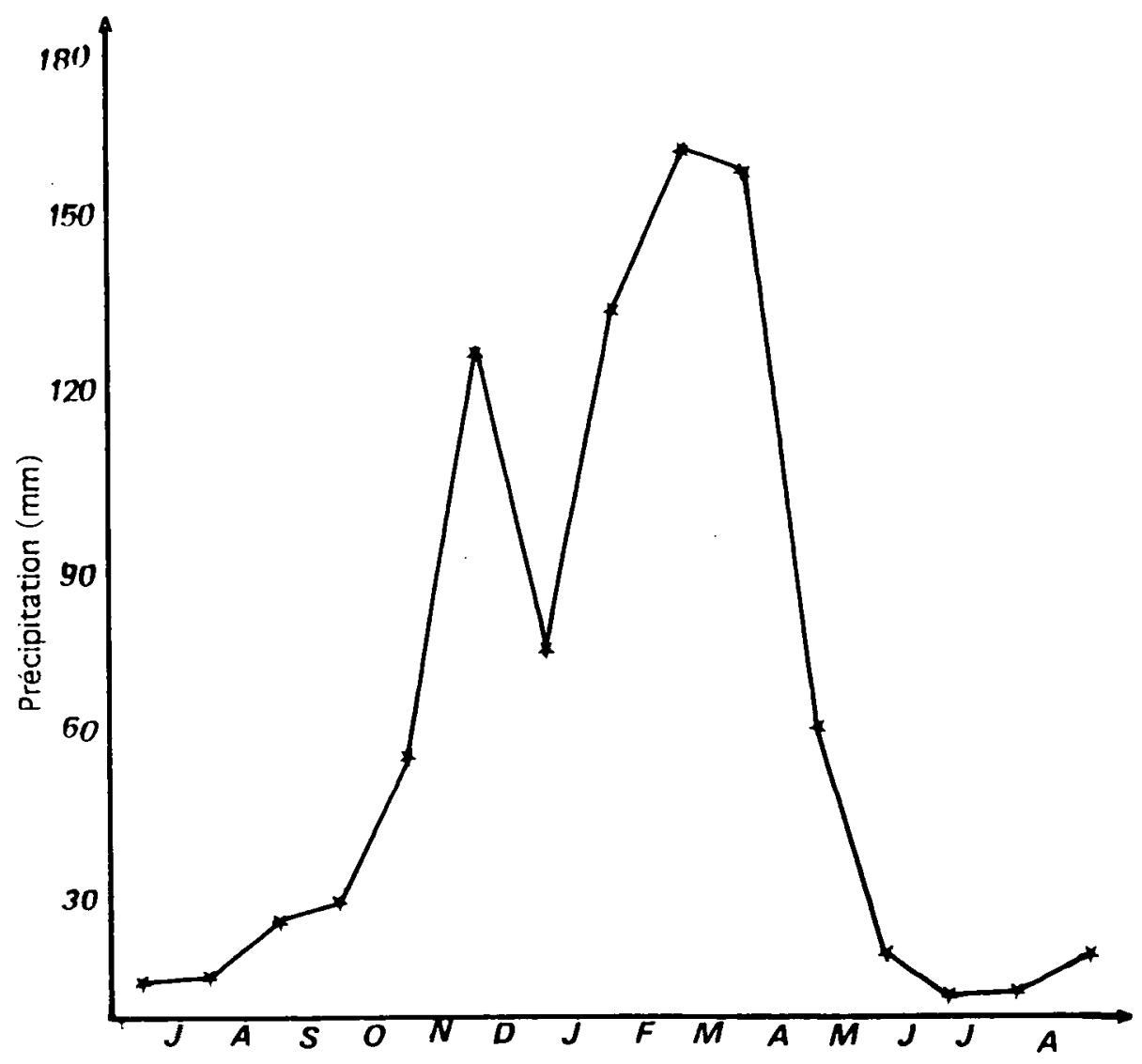

Figure 2 : Schéma illustrant les relations existant entre le régime hydrographique et le régime des pluies.

Figure 2 : Diagram illustrating the relationships existing between the hydrographic regime and the rains regime. 
Traversant des substrats acides, pauvres en substances nutritives assimilables mais riches en acides humiques, les eaux de la Luki et de ses affluents sont, d'après la classification proposée par SACCHI et TESTARD (1971), dystrophes c'est-à-dire pauvres en substances nutritives assimilables mais riches en acides humiques et donc à pH bas.

\section{MATÉRIEL ET MÉTHODES}

Cette étude porte sur 981 poissons récoltés dans le bassin de la rivière Luki dans 6 stations : 4 dans le cours principal $\left(L_{2}, L_{3}, L_{4}\right.$ et $\left.L_{5}\right)$ et 2 dans ces deux affluents $\left(A_{2}\right.$ et $\left.A_{4}\right)$ (Fig. 1).

Les différentes pêches ont été réalisées mensuellement entre juillet 1986 et août 1987, puis trimestriellement entre septembre 1987 et septembre 1988 à l'aide d'un ichtyotoxique local le «Bumi» associé à des filets maillants de vide de maille variant entre 10 et $30 \mathrm{~mm}$. Cet ichtyotoxique était préparé à partir des feuilles et des fruits des arbres suivants : Bremania spatufolia, Cissus araleoides, Dioscorea pterocaulon, Goyera cedrata, Palisota ambigua et Tetrapleura tetraptera.

Pour chacun des poissons examinés, nous avons relevé son sexe, sa longueur standard (LS) (mm), sa masse totale $(\mathrm{M})(\mathrm{g})$, celle de ses gonades $(\mathrm{Mg})$ ainsi que son contenu stomacal. Nous avons aussi examiné les gonades en notant leur aspect général, leur coloration et pour les ovaires, la visibilité des ovocytes à travers la membrane ovarienne des femelles fraîchement capturées.

La taille à la première maturation a été définie comme étant la taille minimale à laquelle on trouve des mâles et des femelles en maturation sexuelle (SRINN KIM YOU, 1976).

Les changements cycliques qui se produisent dans leurs gonades ont été déterminés grâce à l'évolution du rapport gonadosomatique calculé à partir de la relation :

$$
\text { R.G.S. }=100 \times \mathrm{Mg} / \mathrm{M}
$$

liant la masse de gonades (Mg) à celle du poisson (M) (BOUGIS, 1952).

L'échelle de maturité des individus observés a été déterminée à la suite de l'examen macroscopique de leurs gonades. L'échelle adoptée s'inspire de celle utilisée par DURAND et LOUBENS (1970a) ainsi que de celle mise au point par ABOUSSOUAN et LAHAYE (1979).

La fécondité partielle individuelle a été estimée à partir de fragments d'ovaires de 20 à $350 \mathrm{mg}$ pris dans la partie médiane d'un des deux ovaires. Les mesures et les comptages de différents ovocytes ont été faits sous la loupe binoculaire à l'aide d'un micromètre oculaire après leur immersion dans le liquide de Gilson. Pratiquement, nous n'avons considéré que les ovocytes ayant un diamètre supérieur à $100 \mu \mathrm{m}$.

Connaissant le nombre d'ovocytes dans l'échantillon considéré (n), la masse de gonades $(\mathrm{Mg})$ et la masse de l'échantillon $(\mathrm{mg})$, la fécondité absolue $(\mathrm{F})$ a été calculée à partir de la relation $\mathrm{F}=\mathrm{n} \times \mathrm{Mg} / \mathrm{mg}$.

Pour permettre une évaluation approximative de cette fécondité, nous l'avons mise en relation linéaire avec la longueur standard du poisson, sa masse corporelle et celle de sa gonade (PHILIPPART, 1975 ; CONAND, 1979).

L'étude du régime alimentaire a porté sur l'ensemble des contenus stomacaux et intestinaux. Les éléments triés à l'oeil nu, sous la loupe binoculaire et/ou sous microscope étaient séparés, comptés et réunis par catégories. Leur détermination était faite par comparaison avec des spécimens intacts provenant des milieux naturels. L'analyse et la présentation de ces données ont été faites à l'aide de l'indice alimentaire proposé par LAUZANNE (1976) :

$$
\text { I.A.j }=(\% \text { OCj } \times \% \text { Mj }) / 100
$$


où $\% O C$ ou pourcentage d'occurrence de la catégorie $\mathrm{j}$ = (nombre d'estomacs renfermant la catégorie des proies j / nombre d'estomacs renfermant de la nourriture). $\times 100$,

$\% \mathrm{Mj}$ ou volume pondéral = pourcentage en masse de la catégorie $\mathrm{j}$ de proies pour l'ensemble de l'échantillon par rapport à la masse totale de l'ensemble des proies (LAUZANNE, 1976).

La relation liant la masse à la longueur standard a été calculée à partir de l'équation : $M=a L S b$ où a est la constante et $b$ le coefficient de régression représentant la pente de la droite de régression autour de laquelle se regroupent les points représentatifs de la masse en fonction de la longueur standard (LAMOTTE, 1957). La valeur de ce coefficient étant voisin de 3 , nous avons retenu, pour le calcul du coefficient de condition, la formule classique décrite sous la forme $: K=10^{5}\left(\mathrm{M} / \mathrm{LS}^{3}\right)$ (LE CREN, 1951).

Les travaux portant sur la détermination de l'âge des poissons sont extrêmement nombreux èt les techniques utilisées variées. Ces dernières sont généralement rangées en deux grandes catégories: celles qui donnent statistiquement l'âge moyen d'un groupe d'individus et celles qui fournissent un âge individuel (MEUNIER et al., 1979).

L'utilisation de cette dernière technique n'a pas été possible car les marques observées au niveau des écailles et des autres pièces osseuses (rayons osseux et opercules) n'ont pas paru reproductibles. Ce constat nous a conduit à n'estimer que l'âge moyen grâce à la méthode de maximums successifs employée par GHENO et LE GUEN (1968) pour l'étude de la croissance de Sardinella Eba (Val). Celle-ci consiste à partir du premier maximum observable dans une distribution de fréquence et à séparer cette distribution en deux parties $A_{1}$ et $B_{1}$ à gauche et à droite de cette valeur maximalè (Ma). La partie $A_{1}$ située à gauche de $M a$ est une moitié de la distribution normale. En retranchant de la partie $B_{1}$ la partie $A_{1}^{\prime}$ symétrique de $A_{1}$ par rapport à l'axe $L=M a$, òn reconstitue une nouvelle distribution. Si celle-ci est unimodale le problème est résolu, sinon on revient au problème précédent (GHENO et LE GUEN, 1968) (Fig. 3).

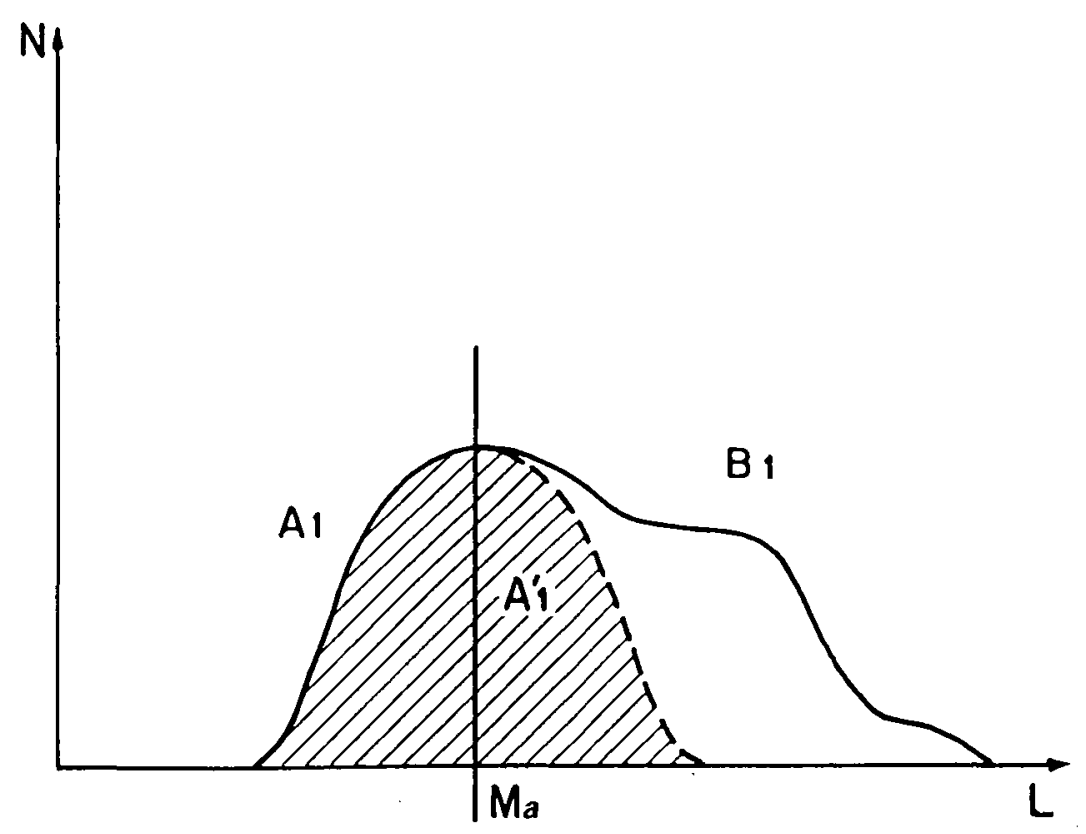

Figure 3 : Principe de décomposition d'une distribution polymodale en distributions unimodales successives (d'après GHENO et LE GUEN, 1968).

Figure 3 : Principle of polymodal distribution decomposition in unimodal successive distributions (from GHENO and LE GUEN, 1968). 
Pour l'estimation de la longévité maximale et de l'âge correspondant aux différents modes observés, nous avons porté sur l'axe des abscisses de l'abaque mis au point par MERONA (1983) la taille maximale moyenne et élevé ensuite une perpendiculaire $(P)$ en ce point. Le nombre de courbes de tailles aux différents âges que coupe $(P)$ donne une estimation de la longévité. Les ordonnées des points d'intersection sont les tailles aux différents âges (MERONA, 1983) (Fig. 4).

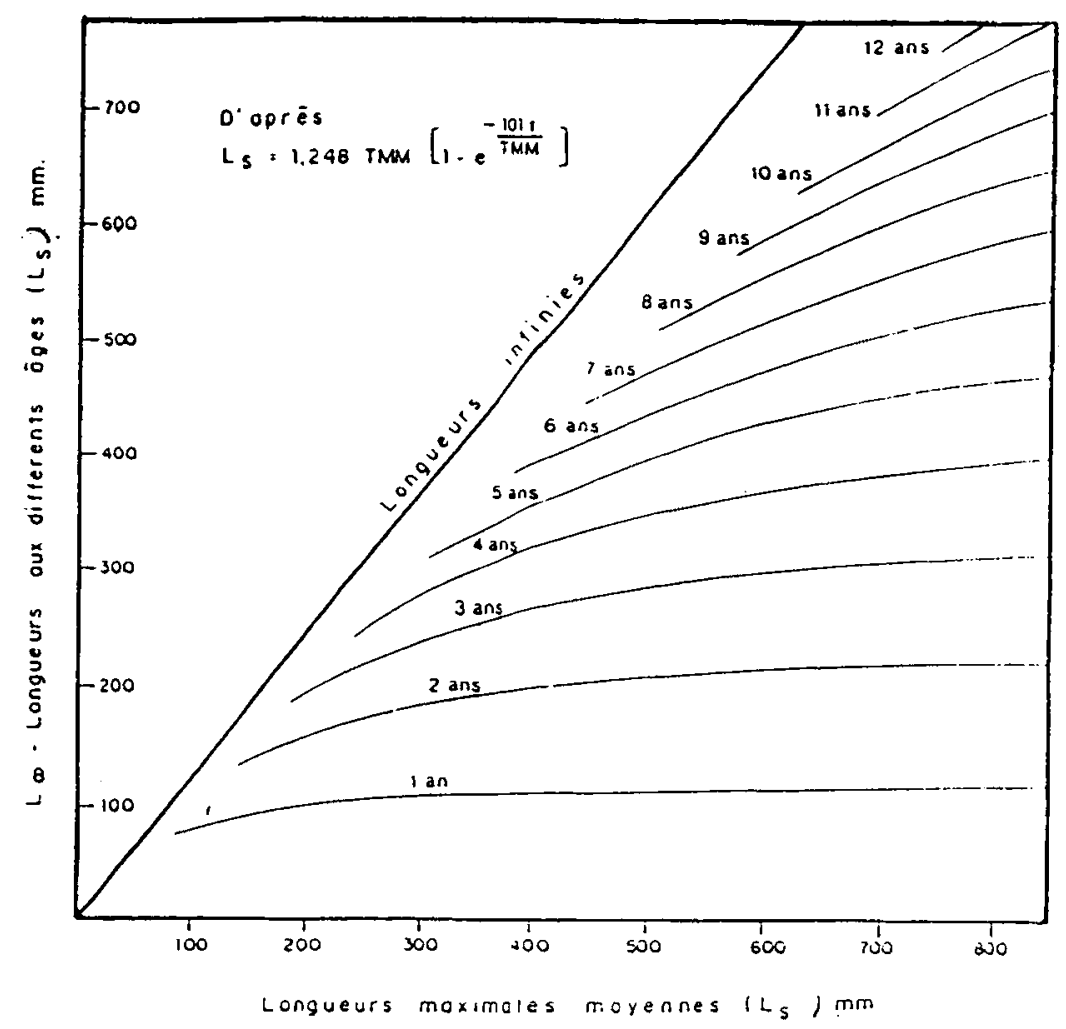

Figure 4 : Abaque des estimations de $L \infty$, LS et longévité en fonction de la taille maximale moyenne (d'après MERONA, 1983).

Figure 4 : Abacus allowing the graphic determination of the length at different ages and the asymptotic length (from MERONA, 1983).

\section{RÉSULTATS}

\section{Sexualité}

En l'absence d'un dimorphisme sexuel externe, le sexe des individus examinés n'a pu être déterminé que par examen des gonades.

Le rapport du nombre des mâles à celui des femelles est de l'ordre de 0,87 . Si on regroupe les individus identifiés par classe de taille de $5 \mathrm{~mm}$, on remarque que les femelles dominent dans les classes inférieures à $52,5 \mathrm{~mm}$ et dans celles comprises entre 82,5 et $102,5 \mathrm{~mm}$ LS (Tab. I).

La taille à la première maturation ( $\left.L_{0}\right)$ est de $37,5 \mathrm{~mm}$ pour les femelles et de $42,5 \mathrm{~mm}$ pour les mâles. Tous les individus sont mûrs $\left(L_{100}\right)$ à partir de $48 \mathrm{~mm}$ (Tab.l).

\section{Reproduction}

\section{Stade de maturation des ovaires}

L'examen macroscopique des gonades fraîches et/ou formolées récoltées durant la période d'étude a permis de reconnaître six stades de développement bien distincts. 
Tableau I : Variation du nombre de mâles et de femelles en fonction de leurs tailles (Ind. = indéterminés).

Table I : Variation of males and females number in accordance with their length (Ind. = indeterminates).

\begin{tabular}{|c|c|c|c|c|}
\cline { 2 - 5 } \multicolumn{1}{c|}{} & \multicolumn{4}{c|}{ Type d'individus } \\
\hline Tailles & Indéterminés & Mâles & Femelles & Mâle/Femelle \\
\hline 12,5 & 160 & & & \\
17,5 & 148 & & & \\
22,5 & 80 & & & \\
27,5 & 67 & & 2 & \\
32,5 & 47 & & 19 & 0,53 \\
37,5 & 60 & 10 & 30 & 0,77 \\
42,5 & 23 & 23 & 16 & 0,94 \\
47,5 & 16 & 15 & 14 & 1,45 \\
52,5 & & 20 & 21 & 1,05 \\
57,5 & & 22 & 23 & 1,07 \\
62,5 & & 25 & 28 & 0,79 \\
77,5 & & 22 & 20 & 0,75 \\
82,5 & & 15 & 15 & 0,40 \\
87,5 & & 6 & 8 & 0,13 \\
92,5 & & 1 & 7 & 0,14 \\
97,5 & & 1 & 1 & 1,00 \\
102,5 & & 1 & & \\
107,5 & & 2 & & \\
117,5 & & 1 & & \\
127,5 & & 2 & & \\
137,5 & & 1 & & \\
142,5 & & 177 & & \\
\hline Total & 601 & & &
\end{tabular}

Stade 0 - Femelle immature : ovaires en forme de bandelettes transparentes et sans vascularisation. Ovocytes et lamelles ovariennes invisibles à l'oeil nu.

Stade I - Femelle en repos sexuel : ovaires peu développés en forme de bandelettes. Ovocytes visibles et enveloppés par un tissu à apparence spongieuse. Lamelles ovariennes présentes et vascularisation externe non marquée.

Stade II - Femelle en maturation : ovaires développés, fermes, peu vascularisés et opaques. Ovocytes bien visibles mais adhérents encore aux lamelles ovariennes.

Stade III - Femelle à maturation avancée : ovaires bien développés, vascularisés et remplis d'ovocytes visibles à l'oeil nu sous la tunica albuginea.

Stade IV - Femelle mûre ou prête à pondre : ovaires envahis d'ovocytes translucides èt libres ainsi que d'autres catégories d'ovocytes décrites précédemment. Ils occupent toute la cavité abdominale et compressent les autres organes internes.

Stade $\mathbf{V}$ - Femelle venant de pondre ou épuisée : ovaires flasques et sanguinolents renfermant quelques ovocytes mûrs et/ou à maturation avancée ainsi que de petits ovocytes en repos ou en voie de maturation. Certains ovaires sont pourvus de corpuscules noirâtres dispersés dans leur masse. Leurs follicules sont flasques, aplatis et déformés.

Échelle de maturation des testicules

Quatre stades de maturation ont été reconnus :

Stade 0 - Mâle immature : les testicules se présentent sous forme de deux filaments minces enrobés de graisse.

Stade I - Mâle adulte en repos sexuel : testicules en forme de bandelettes étroites (0,3 à $1 \mathrm{~mm}$ de largeur); plus ou moins grisâtres et parfois ḅlanchâtres. 
Stade II - Mâle en activité sexuelle : nous avons regroupé dans ce stade les mâles en maturation et mûrs, car leur distinction n'est pas évidente. II y a tous les stades intermédiaires depuis les testicules commençant à se développer jusqu'à des testicules gonflés entièrement blancs à sperme fluide. D'autre part, on ignore à partir de quel moment le sperme est réellement fécondant car son aspect ne change pas au cours de la maturation.

Stade III - Gonades flasques, sanguinolentes, ne renfermant pas de laitance. Ce stade caractérise la fin d'émission des spermatozoïdes.

\section{Cycle annuel des différents stades de maturité}

Diverses techniques permettent de décrire les changements cycliques qui se produisent dans les gonades des poissons : examen macroscopique et microscopique des gonades, apparition des alevins le long des cours d'eau, évolution du rapport gonadosomatique ... (BOELY, 1980 ; KHALAF, 1987). Dans ce travail, nous les avons définis grâce à l'évolution du rapport gonado-somatique. La figure 5 , qui représente cette évolution, montre que le cycle de développement de leurs gonades passe par trois phases : une phase relative à l'arrêt d'activité sexuelle qui s'étend de la deuxième quinzaine de mai pour les femelles et de la deuxième quinzaine de juin pour les mâles à mi-septembre ; une phase de développement et de maturation gonadique qui débute vers mi-septembre et s'arrête en novembre et enfin la phase de reproduction qui commence en décembre et finit vers la première quinzaine de mai pour les femelles et vers la première quinzaine de juin pour les mâles.

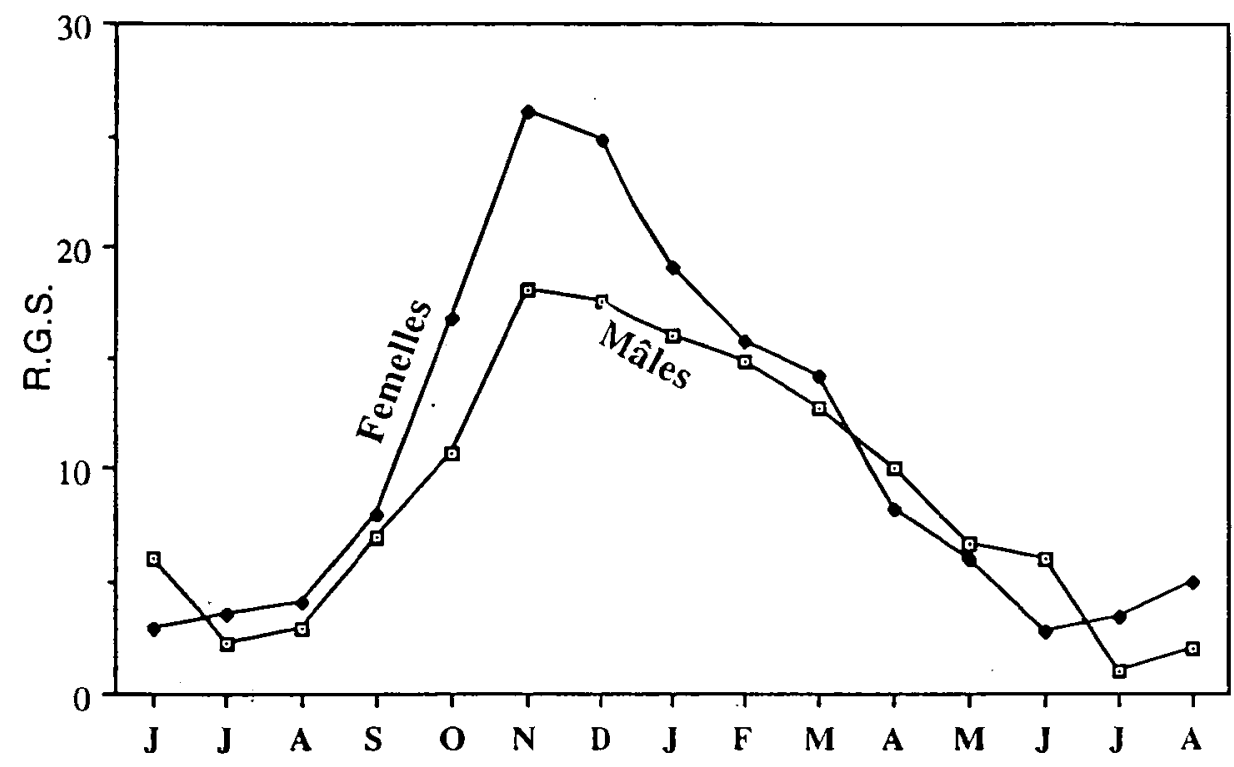

Figure 5 : Variation mensuelle du rapport gonado-somatique chez $B$. holotaenia mâles et femelles.

Figure 5 : Monthly variation of gonadosomatic indice of $B$. holotaenia males and females.

La présence de femelles à rapport gonado-somatique élevé (Fig. 5), à maturation avancée (Fig. 6) ainsi que l'apparition d'alevins dans les différents cours d'eau durant toute la saison des pluies, a conduit à supposer que Barbus holotaenia se reproduit à cette époque.

\section{Fécondité}

Elle a été déterminée en comptant dans les ovaires de 56 femelles le nombre d'ovocytes ayant un diamètre supérieur à $100 \mu \mathrm{m}$. Les valeurs obtenues varient d'une femelle à une autre, la différence étant parfois du simple au quintuple (Tab. II). 

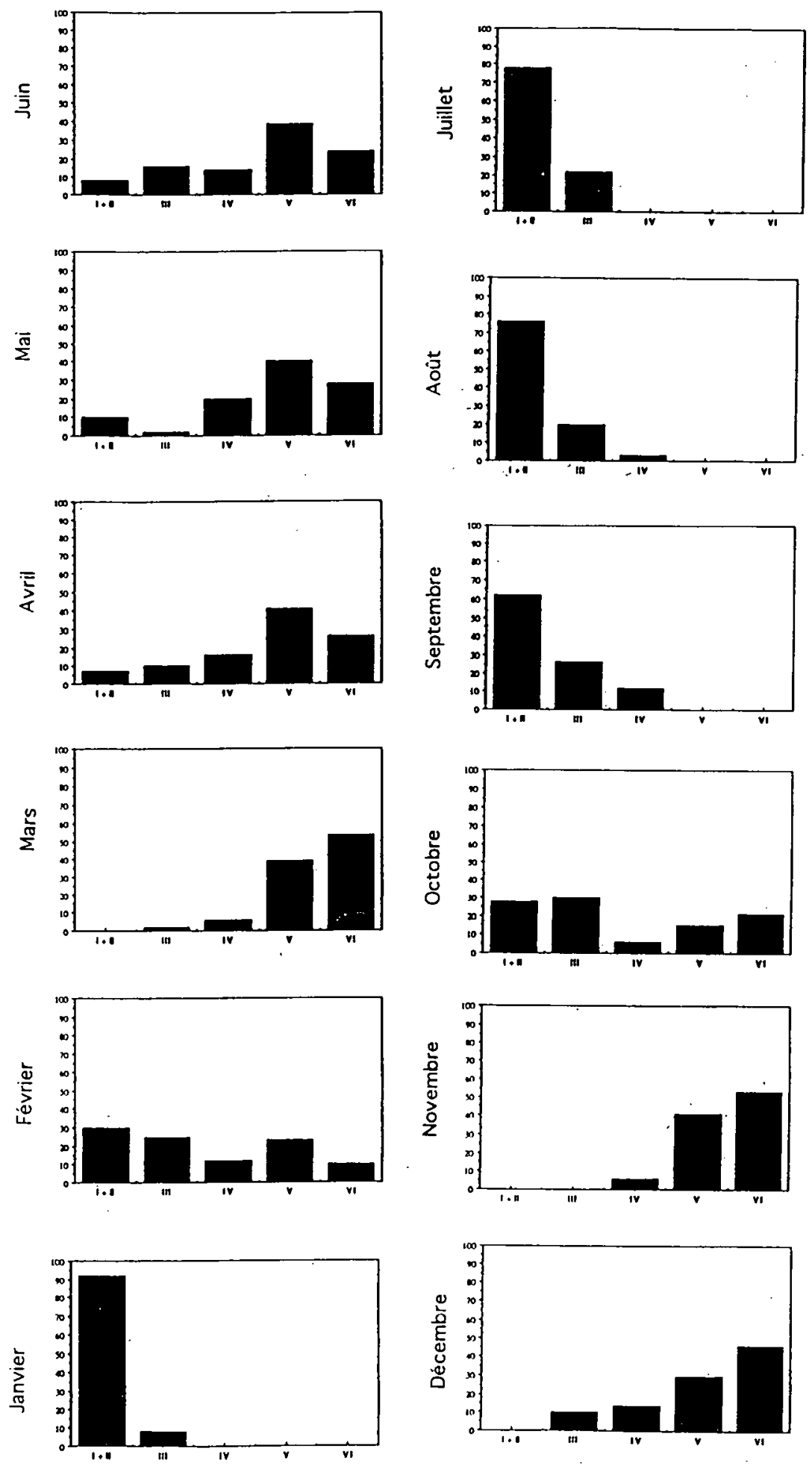

Figure 6 : Pourcentage mensuel de différents stades de maturité chez les femelles de B. holotaenia.

Figure 6 : Monthly percentage of different stages of maturity in $B$. holotaenia females. 
Tableau II : Valeurs maximales, moyennes et minimales de la fécondité par classe de taille (A), de masse totale (B) et de masse de gonade (C).

Table II : Maximum, mean and minimum values of fecundity by length (A), total weight (B) and gonad weight classis (C).

A
\begin{tabular}{|lllll|}
\hline $\begin{array}{l}\text { Ls médianes } \\
\text { des classes } \\
(\mathrm{mm})\end{array}$ & $\mathrm{N}$ & Féc. maxi. & Féc.moy. & Féc.min. \\
\hline 47,5 & 1 & 1039 & - & - \\
52,5 & 3 & 776 & 542 & 127 \\
57,5 & 3 & 1906 & 1134 & 362 \\
62,5 & 15 & 1873 & 644 & 120 \\
67,5 & 13 & 1248 & 653 & 112 \\
72,5 & 12 & 2244 & 760 & 191 \\
77,5 & 4 & 1136 & 720 & 509 \\
82,5 & 3 & 2260 & 1462 & 961 \\
87,5 & 2 & 750 & 750 & 750 \\
92,5 & 3 & 1486 & 1486 & 1486 \\
97,5 & 1 & 920 & - & - \\
\hline
\end{tabular}

B
\begin{tabular}{|lllll|}
\hline $\begin{array}{l}\text { M médianes } \\
\text { des classes } \\
(\mathrm{g})\end{array}$ & $\mathrm{N}$ & Féc. maxi. & Féc.moy. & Féc.min. \\
\hline 3,25 & 1 & & & \\
4,25 & 6 & 723 & & \\
5,25 & 8 & 1248 & 587 & 193 \\
5,75 & 2 & 1906 & 961 & 127 \\
6,25 & 12 & 760 & 561 & 362 \\
7,25 & 4 & 2244 & 694 & 112 \\
8,25 & 4 & 786 & 544 & 120 \\
8,75 & 1 & 962 & 586 & 254 \\
9,25 & 3 & 876 & - & - \\
10,25 & 8 & 884 & 507 & 294 \\
11,25 & 2 & 1289 & 774 & 340 \\
15,75 & 3 & 2260 & 1713 & 1165 \\
24,25 & 1 & 961 & 732 & 674 \\
25,25 & 1 & 920 & - & - \\
\hline
\end{tabular}

\begin{tabular}{l} 
C \\
\begin{tabular}{|lllll|}
\hline $\begin{array}{l}\text { m médianes } \\
\text { des classes } \\
(\mathrm{mg})\end{array}$ & $\mathrm{N}$ & Féc. maxi. & Féc.moy. & Féc.min \\
\hline 2,5 & 2 & 750 & & \\
7,5 & 26 & 1173 & 543 & 135 \\
12,5 & 21 & 1906 & 782 & 112 \\
17,5 & 2 & 1289 & 1083 & 876 \\
22,5 & 3 & 2244 & 1868 & 1486 \\
27,5 & 1 & 1039 & - & - \\
32,5 & 1 & 2260 & - & - \\
\hline
\end{tabular} \\
\hline
\end{tabular}


Cette fécondité augmente en fonction de la longueur standard de la femelle (LS), de sa masse totale $(\mathrm{Mg})$ et de celle de ses gonades $(\mathrm{mg})$ suivant les équations de régression suivantes :

$$
\begin{array}{ll}
F=9,4 L S-503,57 & (n=56 ; r=0,35) \\
F=51,92 M g+295 & (n=56 ; r=0,51) \\
F=628,7 m g+45,35 & (n=56 ; r=0,86)
\end{array}
$$

La fécondité relative correspondant au nombre d'ovocytes produits par une unité de masse totale de la femelle (BAGENAL, 1973 ; WOOTTON, 1979 ; KARTAS et QUIGNARD, 1984) varie entre 1994 et 15920 ovocytes.

\section{Régime alimentaire}

II varie en fonction de l'âge des poissons. Les jeunes se nourrissent presque exclusivement de cladocères et de quelques larves de chironomides.

Le régime alimentaire des adultes varie en fonction de la saison. En saison des pluies, il est constitué d'invertébrés aquatiques (essentiellement des larves de chironomides, d'éphémeroptères, de diptères, de trichoptères et de petits coléoptères), d'insectes terrestres entraînés par l'eau de ruissellement et de quelques crevettes (Cardina africana et Desmocaris trispinosa). En saison sèche, ils se nourrissent presque exclusivement de crevettes, d'écailles et d'hydracariens. On trouve également dans les estomacs des grains de sables probablement ingérés avec les invertébrés benthiques (Fig. 7).

\section{Relation longueur standard / masse}

La relation liant la masse à la longueur standard de ce poisson est la suivante : $M=2,29$ LS $^{2,99}(r=0,95)$.

Le calcul direct des valeurs individuelles du coefficient de condition $\mathrm{K}$ a donné les résultats groupés dans le tableau III.

II n'y a pas de corrélation entre ce paramètre et la taille de ces poissons $(r=0,036)$. Le test $t$ de Student a encore montré qu'il n'y a pas de différence significative de coefficient de condition moyen entre les individus de deux sexes $(2,40$ pour les mâles et 2,46 pour les femelles) ni même entre les adultes et les immatures.

Les valeurs observées en saison des pluies $(3,22$ pour les mâles et 4,17 pour les femelles). sont significativement différentes de celles enregistrées en saison sèche $(1,99$ pour les mâles et 1,77 pour les femelles) $(t>2,60)$.

\section{Croissance}

La lecture des écailles, des opercules et des coupes des rayons osseux de nageoires pectorales a montré l'existence des arrêts de croissance irréguliers et non reproductibles. La méthode de rétro-calcul était donc inapplicable. L'histogramme de fréquence de tailles établi à partir de la méthode de maximums successifs a été seul utilisé pour l'évaluation de la croissance (Fig. 8).

La longévité maximale estimée à partir de l'abaque mis au point par MERONA (1983) est de 15 mois environ. Lé groupe d'âge correspondant aux différents pics de la distribution polymodale est de $0^{+}, 3^{+}, 6^{+}, 12^{+}, 15^{+}$mois.

\section{CONCLUSION ET DISCUSSION}

Les résultats de cette étude montrent que Barbus holotaenia a une longévité assez courte et une reproduction bien étalée en saison des pluies. Ce dernier phénomène a été également signalé chez B. sublineatus (Daget, 1954) ; B. trispilos (Bleeker, 1863) et chez B. ablabes (Bleeker, 1863a) de Côte-d'Ivoire par ALBARET (1982) et semble étayer l'hypothèse, selon laquelle les précipitations qui entraînent des crues fortes et durables favorisent un bon recrutement alors qu'inversement, une crue annuelle déficitaire entraine des conséquences néfastes sur les stocks (ALBARET, 1982). Cette simultanéité résulterait 

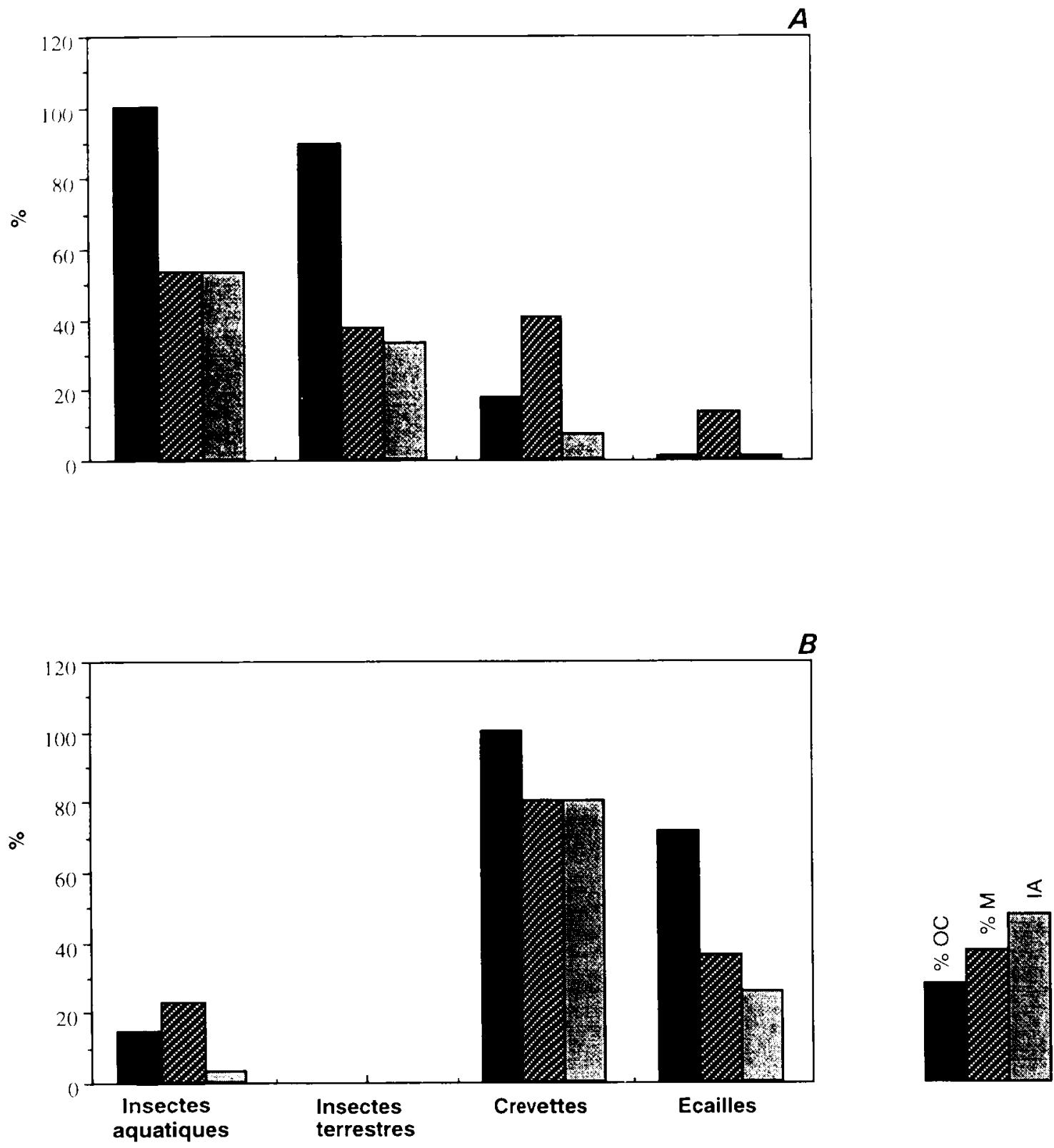

Figure 7 : Répartition d'aliments consommés par $B$. holotaenia en fonction de la saison $A$ : saison des pluies, $B$ : saison sèche $(\% O C=$ pourcentage d'occurrence, $\% M=$ volume pondéral, $I A=$ indice alimentaire).

Figure 7 : Distribution of foods consumed by $B$. holotaenia in relationship with the season $A=$ rains season, $B=$ dry season $(\% O C=$ occurrence percentage, $\% M=$ weight percentage, $\mid A=$ alimentary indice $)$.

probablement de l'adaptation du cycle reproducteur des poissons sur le cycle général des eaux, puisque c'est en cette saison que les disponibilités de nourriture pour l'élaboration des produits sexuels et pour l'alimentation des jeunes alevins sont maximales.

Le sex ratio est inférieur à 1. Ce phénomène pourrait être dû au fait que les mâles immatures, difficiles à identifier, auraient été, dans la plupart des cas, classés dans la série des indéterminés ou alors au fait que dans la nature les femelles sont globalement plus nombreuses que les mâles. 
Tableau III : Relation entre le coefficient $\mathrm{K}$ et la longueur standard chez B. holotaenia (fk = fréquence totale).

Table III : Relationship between the condition coefficient and the standard length of $B$. holotaenia (fk = total frequency).

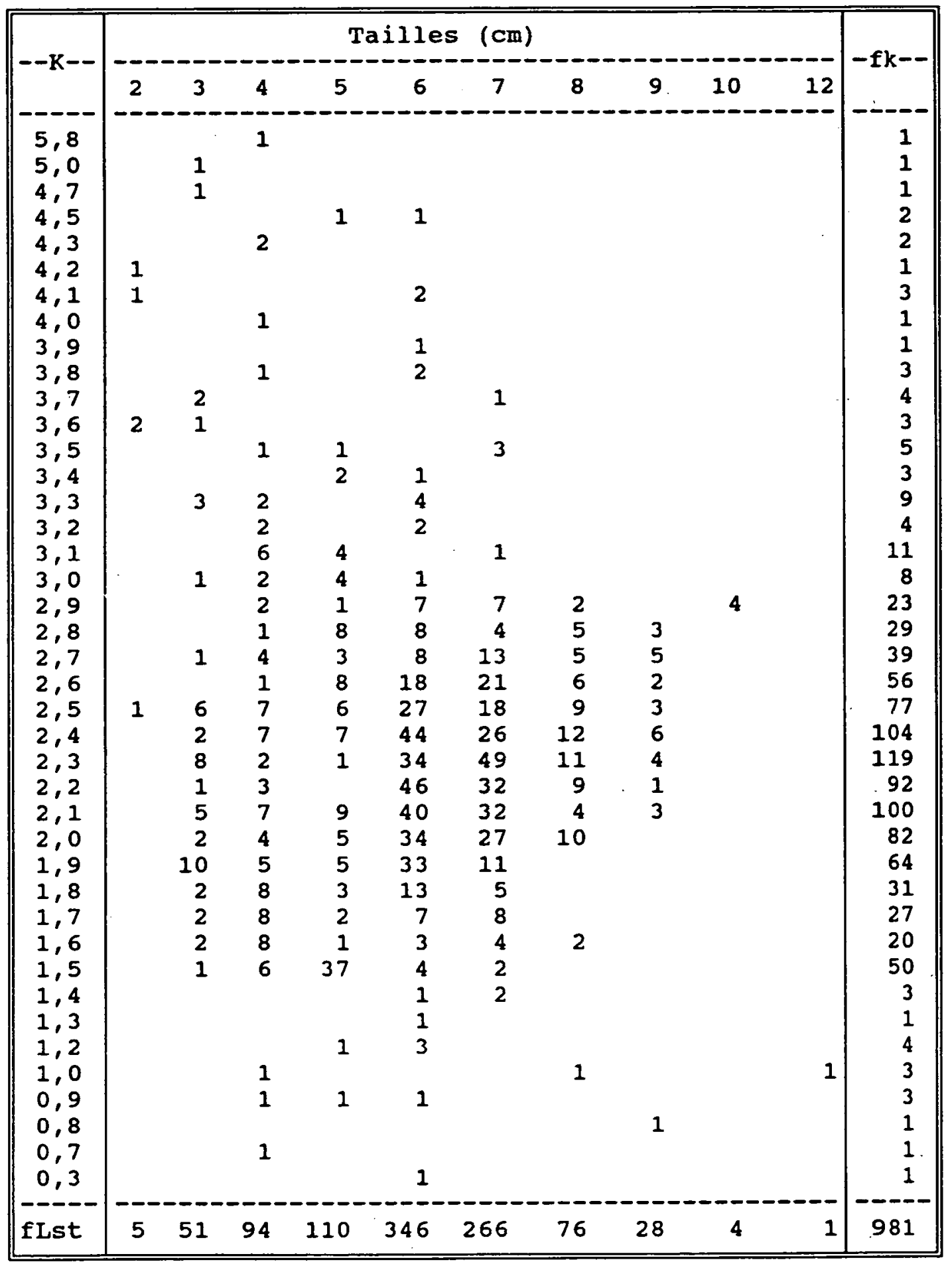




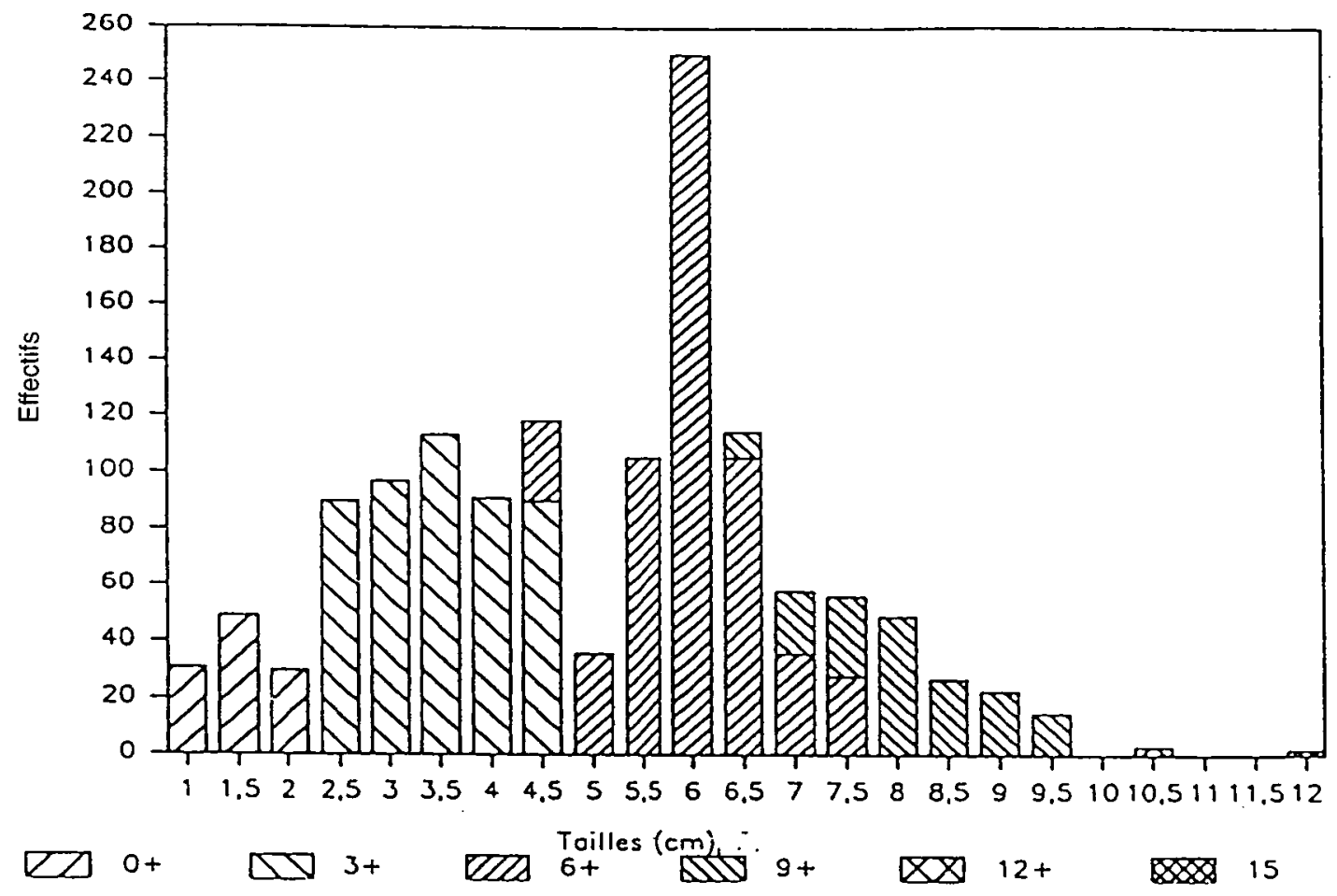

Figure 8 : Histogramme de fréquences de tailles de $B$. holotaenia avec
décomposition en six distributions successives.

Figure 8 : Length frequency histogram of $B$. holotaenia with decomposition in six successive distributions.

B. holotaenia est un entomophage. Cette observation est conforme à celles faites par GOSSE (1963) chez les Barbus vivant dans la région de Yangambi et par MATTHES (1964) chez ceux du lac Tumba et de la région d'Ikela.

Le coefficient de condition est plus faible en saison sèche qu'en saison des pluies. L'accumulation des réserves destinées à la formation des gonades, durant cette période, ne semble pas être responsable de l'élévation de ce coefficient car les immatures suivent le même schéma que les adultes. A la suite de leur travail sur Alestes baremoze, DURAND et LOUBENS (1970b) ont montré que la maturation avancée des gonades ne modifie pas le coefficient de condition des poissons, il y a simple transfert de matière depuis les réserves de la cavité abdominale et des muscles vers les gonades sans variation sensible de la masse totale des individus. Ceci permet de supposer que l'augmentation de ce coefficient pendant la saison des pluies serait directement liée à l'intensification des activités trophiques ainsi qu'au retour probable des poissons dans leur aire de nourrissage. En saison sèche, ils puisent dans leurs réserves de quoi assurer leur croissance en longueur et leurs dépenses énergétiques.

Ce coefficient est indépendant de la taille des poissons étudiés car la valeur de $r$ est très faible $(r=0,036)$ et, par conséquent, non significativement différente de 0 . Ce type d'observation a été réalisé par DAGET (1964) chez Lates niloticus de la région de Mopti et par MOREAU (1971) chez Tilapia rendalli des lacs Itasy et Mantasoa (Madagascar).

Le coefficient d'allométrie $(2,99)$ étant voisin de la valeur théorique $(3)$, nous avons supposé que Barbus holotaenia ne change pas de forme au cours de sa croissance. Les variabilités individuelles observées proviennent soit de la disponibilité de la nourriture soit de l'état de maturité des gonades, notamment chez les femelles (BAUCHOT et BAUCHOT, 1978). Ces observations se rapprochent sensiblement de celles faites par DAGET et ILTIS (1965), DURAND et LOUBENS (1970b) ainsi que de celles faites par DURAND et al. (1973) chez bon nombre de poissons d'eau douce africains. 


\section{BIBLIOGRAPHIE}

ABOUSSOUAN A., LAHAYE J., 1979. Les potentialités des populations ichtyologiques. Fécondité et ichtyoplancton. Cybium, 3 (6), 29-46.

ALBARET J.J., 1982. Reproduction et fécondité des poissons d'eau douce de Côted'Ivoire. Rev. Hydrobiol. Trop., 15 (4), 347-371.

AUBREVILLE A., 1949. Climats, forêts et désertification de l'Afrique Tropicale. Soc. éd. géo., mar., col., $351 \mathrm{p}$.

BAGENAL T.B., 1973. Fish fecundity and its relation with stock and recruitment. Rapp. P.V. Réun. Cons. Perm. Inter. Exploit. Mer., 164, 186-198.

BANISTER K.E., 1973. A revision of the large Barbus (Pisces, Cyprinidae) of East and Central Africa. Studies on African Cyprinidae. Part II. Bull. Brit. Mus. (Nat. Hist.) Zool., 26 (1), 1-148.

BAUCHOT R.L., BAUCHOT M.L., 1978. Coefficient de condition et indice pondéral chez les téléostéens. Cybium 3॰ Sér., 3-16.

BLACHE J., 1964. Les poissons du bassin du Tchad et du bassin adjacent du Mayo Kebbi. Étude systématique et biologique. Mém. ORSTOM, 4 (2), 485 p.

BOELY T., 1980. Étude du cycle sexuel de la sardinelle ronde (Sardinella aurita Val., 1847) au Sénégal. Cah. ORSTOM, Sér. Océanogr. Trop., 17 (1), 3-13.

BOUGIS P.; 1952. Recherches biométriques sur les rouges (Mullus barbatus L., Mullus surmuletus). Thèse doct. Univ. de Paris, 174.

BOULENGER G.A., 1909-1916. Catalogue of the freshwater fishes of Africa in the British Museum of Natural History. London, Vol. II, $513 \mathrm{p}$.

CHECK LIST OF THE FRESHWATER FISHES OF AFRICA, 1984. In DAGET J., GOSSE J.P., THYS VAN DEN AUDENAERDE, MARC, ORSTOM; Tome 1, $410 \mathrm{p}$.

CONAND C., 1979. Données complémentaires sur le cycle sexuel et variations interannuelles de la fécondité du Tassergal de la côte nord du Sénégal. Bul. I.F.A.N., Tome 38, sér. A, $n^{\circ} 4,900-920$.

CUGNY P., MUTAMBUE S., 1991. Analyse des données multidimensionnelles appliquées à l'étude de la distribution de la faune piscicole du bassin de la Luki (sous-affluent du fleuve Zaïre). (Zaïre), Rev. Mus. Hist. nat. Toulouse, 21-27.

DAGET J., 1961. Poissons du Niari-Kouilou récoltés par MM. CH. ROUX, J. DUCROZ et J.P. TROADEC (Afrique Noire, Région Gabon-Congo). Bull. Mus. nath. hist. nat. Paris, (2), 33 ( 6), 577-586.

DAGET J., 1964. Notes sur le Lates niloticus (Poissons Centropomidae) immature de la région de Mopti. Bull. I.F.A.N., Sér. A 26, $n^{\circ} 4$, 1520-1539.

DAGET J., ILTIS A., 1965. Poissons de Côte-d'lvoire (eaux douces et saumâtres). Mém. I.F.A.N., $n^{\circ} 74,385$ p.

DURAND J.R., LOUBENS G., 1970a. Observations sur la sexualité et la reproduction des Alestes baremoze du bas Chari et du lac Tchad. Cah. ORSTOM, sér. Hydrobiol., Vol. IV, $n^{\circ} 2,61-81$.

DURAND J.R., LOUBENS G., 1970b. Variation du coefficient de condition chez Alestes baremoze (Pisces, Characidae) du bas Chari et du lac Tchad. Cah. ORSTOM, sér. Hydrobiol., 4 (1), 27-44.

DURAND J.R., FRANC J., LOUBENS G., 1973. Clefs longueurs-poids pour 58 espèces de poissons du bassin du lac Tchad. ORSTOM N'Djemena, 35 p. multigr.

FOWLER H.W., 1930. The freshwater fishes obtained by the GRAY African expedition 1929. With notes on other species in the Academy collection, Proc. Acad. Sci. nat. Hist. Philad., 82, 27-83. 
GERY J., 1965. Poissons du bassin de l'Ivondo. Biol. Gabon, 1, 375-393.

GHENO Y., LE GUEN J.C., 1968. Détermination de l'âge et croissance de Sardinella eba (Val) dans la région de Pointe Noire. Cah. ORSTOM, Sér. Océanogr., vol. VI, $n^{\circ} 2$, 69-82.

GOSSE J.P., 1963. Le milieu aquatique et écologie des poissons dans la région de Yangambi. Annls. Mus. Roy. Afr. Centr., Sér. in $8^{\circ}$ Sc. Zool., $n^{\circ} 116,115-247$.

KARTAS F., QUIGNARD J.P., 1984. La fécondité des poissons téléostéens. Masson, Paris, $121 \mathrm{p}$.

KHALAF G., 1987. Le cycle sexuel de Capoeta damascina (Cyprinidae) dans les cours d'eau libanais. Cybium, vol. 11, $n^{\circ} 4,395-402$.

LAMOTTE M., 1957. Initiation aux méthodes statistiques en biologie. Masson \& Cie, Paris, $144 \mathrm{p}$.

LAUZANNE L., 1976. Régimes alimentaires et relations trophiques des poissons du lac Tchad. Cah. ORSTOM, sér. Hydrobiol., Vol. X, $n^{\circ} 4,267-310$.

LE CREN E.D., 1951. The length-weight relationship and seasonal cycle in gonad weight and condition in perch. J. animal Ecol., 20, 2, 201-219.

LEFEUVRE J.C., RAFFIN J.P., BEAUFORT F., 1979. Protection, conservation de la nature et développement. In Ecologie et Développement. Les connaissances scientifiques, écologiques et développement et la gestion des ressources, et de l'espace. Mins. Env. / INERA. Ed. CNRS, 31-98.

MAHNERT V., GERY J., 1982. Poissons du bassin de I'lvindo. IX. Notes sur le genre Barbus (Cyprinidae). Rev. Suis. Zool. Ann/s. Soc. Suis. Zool. et du Mus. Hist. Nat. Genève, Tome 89, Fasc. 2, 461-495.

MATTHES H., 1964. Les poissons du lac Tumba et de la région d'lkela. Etude systématique et écologique. Annis. Mus. Roy. Afr. Centr, Sér. in $8^{\circ}$ Sc. Zool., $n^{\circ} 126,203$ p.

MERONA B. de, 1983. Modèle d'estimation rapide de la croissance des poissons. Application aux poissons d'eau douce d'Afrique. Rev. Hydrobiol. Trop., 16 (1), 103-113.

MEUNIER F.J., PASCAL M., LOUBENS G., 1979. Comparaison de méthodes squelettochronologiques et considérations fonctionnelles sur le tissu osseux acellulaire d'un Osteichtyen du Lagon néocalédonien. Aquaculture, 17, 137-157.

MOREAU J., 1971. Biologie comparée de Tilapia rendalli (Boulenger) (Pisces, Cichlidae) au lac Itasy et au lac Mantasoa. Cah. ORSTOM, sér. Hydrobiol, vol. V, n 1, 3-52.

MUTAMBUE S., 1984. Contribution à l'étude de l'écologie de la rivière Luki (sous-affluent du fleuve Zaïre) : Bassin versant - Poissons. Thèse doct. $3^{e}$ Cycle, UPS/Toulouse, $214 \mathrm{p}$.

MUTAMBUE S., 1985. Premières données sur la faune piscicole de la rivière Luki. Cybium, $9(1), 17-28$.

MUTAMBUE S., 1992. Le bassin de la Luki (Zaïre) et son aménagement. Systématique, Biologie et Ecologie de sa faune piscicole. Thèse doct. UPS/Toulouse, $325 \mathrm{p}$.

PHILIPPART J.C., 1975. Dynamique des populations de poissons d'eau douce non exploités. In “Problèmes d'Ecologie», LAMOTTE et BOURLIERE (eds), Paris, 292-395.

POLL M., 1941. Etude systématique et morphologique d'une collection des poissons de l'Uele (Congo belge) comprenant 3 espèces nouvelles. Bull. Mus. Roy. Afr. Centr. Belg., 17 (50), 1-18.

POLL M., 1960. Recherches sur la faune ichtyologique de la région du Stanley-Pool. Annls. Mus. Roy. Congo belge, in 8', Zool., vol. 71, 75-174.

POLL M., 1967. Contribution à la faune ichtyologique de l'Angola. Publoes cult. Com. Diam. Angola, Mus. Dundo, $n^{\circ} 75,381 \mathrm{p}$. 
POLL M., GOSSE J.P., 1963. Contribution à l'étude systématique de la faune ichtyologique du Congo Central. Annls. Mus. Roy. Afr. Centr., Sér. in 8 ${ }^{\circ} \mathrm{n}^{\circ} 116,44-110$.

ROMAN R., 1971. Peces de Rio Muni, Guinea ecuatorial (Aguas dulces y salobres). Barcelona, $295 \mathrm{p}$.

SACCHI C.F., TESTARD P., 1971. Ecologie animale, organisme et milieu. Doin (ed.), 480 p.

SRINN KIM YOU, 1976. Biologie d'Hydrocinus forskalii (Pisces, Characidae) du bassin tchadien. Thèse doct. $3^{\circ}$ Cycle, UPS/Toulouse, $126 \mathrm{p}$.

WOOTTON R.J., 1979. Energy costs of eggs production and environment determinant of fecundity in teleost fishes. In MILLER P.J., Fish phenology : anabolic adaptiveness in teleost. Symp. Zool. Soc. Lond., 44, 133-159. 